\title{
Les structures de participation de la micro- culture de classe dans une leçon de mathématiques
}

\section{Lucie Mottier Lopez}

S'appuyant sur les propositions théoriques des perspectives situées, l'article expose l'étude détaillée d'une leçon de mathématiques en quatrième année primaire dont le déroulement est structuré par différentes organisations sociales. Le but est d'interroger le plan communautaire de la classe à partir de l'analyse des processus interpersonnels entre les élèves et avec l'enseignant en examinant la co-élaboration de l'interprétation et du raisonnement mathématiques relativement à un problème multiplicatif. Les résultats de recherche montrent qu'une même norme représentative des structures de participation valorisées dans la microculture de classe peut structurer les interactions didactiques à la fois dans l'interaction collective et dans les travaux de groupes des élèves. D'autre part, l'actualisation de cette norme dans des formes contrastées de co-élaboration entre élèves est mise en évidence.

\section{Introduction}

La présente étude s'inscrit dans une perspective située de la cognition et de l'apprentissage ${ }^{1}$ qui considère que la situation co-produit les connaissances à travers l'activité qui prend place dans la situation (Brown, Collins \& Duiguid, 1989). Le contexte n'est plus vu comme étant seulement l'environnement dans lequel se produit l'activité, mais il devient une partie intégrale de l'activité, avec ses systèmes interactifs et sociaux, le matériel et les ressources représentationnelles utilisés par les individus (Greeno, 1997). Parmi ces dimensions contextuelles, il a été choisi d'examiner plus spécifiquement les systèmes d'attentes et d'obligations qui structurent les interactions didactiques dans l'enseignement et l'apprentissage des mathématiques. Sur la base de la distinction définie par Rogoff (1995) entre les plans individuel, interpersonnel et communautaire, la recherche se focalise en premier lieu sur la dimension interpersonnelle de l'activité mathématique co-élaborée en classe. Le but est de tenter d'interroger ce qui relève du plan communautaire à partir de l'analyse des processus interpersonnels entre les élèves et avec l'enseignant, en examinant notamment le développement de l'interpréta- 
tion et du raisonnement mathématiques au cours des activités conjointes. Le raisonnement est vu ici comme non seulement une caractéristique individuelle mais aussi comme une activité sociale de l'individu avec autrui (Minick, 1985, cité par Moll \& Whitmore, 1993; Resnick, Pontecorvo \& Säljo, 1997).

Suite à une brève présentation théorique, l'étude d'une leçon de mathématiques dans une classe de quatrième année primaire est exposée. ${ }^{2}$ Différentes organisations sociales ont structuré le déroulement de la leçon: un travail en dyades, un temps d'interactions semi-collectives menées par l'enseignant et une reprise du travail en dyades. Les résultats de recherche présentent plus particulièrement la démarche de résolution de deux dyades, relativement à un problème multiplicatif issu des moyens d'enseignement romands des mathématiques de quatrième année primaire (Danalet, Dumas, Studer \& Villars-Kneubühler, 1998). Une brève discussion finale interroge l'intérêt d'étudier les structures de participation de la communauté classe, afin de promouvoir des situations authentiques d'enseignement et d'apprentissage à l'école.

\section{Problématique}

\section{Perspectives situées de la cognition et de}

L'apprentissage

Les résultats de recherches anthropologiques mettent en évidence des points de rupture entre l'apprentissage scolaire et la nature de l'activité cognitive dans les situations extrascolaires, alors qu'il est constaté, par exemple, que les individus n'utilisent pas dans des situations de la vie quotidienne les algorithmes et les règles mathématiques apprises à l'école (e.g., Lave, 1988 ; Resnick, 1987). Ce constat incite, d'une part, à questionner l'efficacité des situations d'enseignement et d'apprentissage scolaires, et, d'autre part, à développer une réflexion théorique sur l'influence du caractère routinier de l'activité, ainsi que des informations et significations configurées dans les objets et les contraintes de la situation.

Développant une conceptualisation de la situation et de l'activité qui attribue une nature intrinsèquement située aux processus d'enseignement et d'apprentissage, Lave et Wenger (1991) théorisent l'apprentissage comme un acte de participation à des pratiques sociales propres à une ou plusieurs communautés reliées. Comme le soulignent Baeriswyl et Thévenaz (2001), l'étude des processus participatifs dans lesquels prennent place à la fois les processus et les produits de l'apprentissage, cherche dès lors à intégrer les contraintes et les conditions dans lesquelles ils se développent. Les aspects contextuels, tels que les ressources matérielles et représentationnelles dans la situation, les modalités d'interaction, les systèmes de significations ainsi que la structuration temporelle et spatiale des activités semblent d'autant plus importants à étudier dans le contexte scolaire qu'ils participent non seulement à la co-élaboration de l'activité, mais qu'ils re- 
présentent une part de ce que l'élève apprend, au même titre que le contenu académique visé par la tâche (Allal et al., 2001). Lave (1988) propose de distinguer les aspects contextuels qui relèvent du "donné» de la situation (consignes, outils, organisation matérielle et sociale, ...) de ceux qui se construisent dans l'interaction continue entre les participants (les significations, les normes, les structures de participation, ....). C'est dans cette dernière dimension que s'inscrit l'objet d'étude de notre recherche, à savoir les systèmes d'attentes et d'obligations tels qu'ils se négocient sur le plan interpersonnel, afin d'interroger ensuite les structures de participation de la communauté classe.

\section{Les normes de la microculture de la classe}

Prenant appui sur les propositions théoriques des perspectives situées, Cobb et al. (1997) proposent un cadre analytique et interprétatif de la microculture de classe, avec la définition de différentes dimensions servant à caractériser les processus de participation, dont notamment les normes rattachées aux structures de participation valorisées sur le plan communautaire. Faisant référence aux travaux de Florio (1978) et d'Erickson et Schulz (1981), Lampert (1990) définit la notion de structure de participation comme étant l'attribution des droits et des obligations aux personnes participant à des événements sociaux:

It [les structures de participation] represents the consensual expectations of the participants about what they are supposed to be doing together, their relative rights and duties in accomplishing tasks, and the range of behaviors appropriate within the events (Lampert, 1990, p. 34).

Dans le contexte scolaire, les structures de participation définissent les rôles et les systèmes d'attentes et d'obligations à la fois de l'enseignant et des élèves en relation avec les activités d'enseignement et d'apprentissage. Notons ici le rapprochement entre cette conception et la notion de contrat didactique, issue du courant francophone de la didactique des mathématiques, qui cherche à saisir le processus de négociation constant et dynamique du sens et de la définition des rôles et des obligations de chacun relatifs au contenu mathématique (Brousseau, 1986; Schubauer-Leoni, 1986). De même, le concept de microculture de classe pourrait être relié au construit théorique de coutume de classe, tel que développé par Balacheff (1988), qui cherche à saisir les ensembles de pratiques obligatoires dans la classe vue comme une société coutumière, ainsi que les façons d'agir établies par l'usage. Toutefois, il conviendrait de théoriser plus précisément les liens entre ces différentes notions et leur registre d'application, une comparaison par ailleurs proposée par Balacheff entre la notion de contrat didactique et celle de coutume de classe.

Une recherche de Yackel et Cobb (1996), dans le cadre d'une classe de deuxième année primaire qui cherchait à promouvoir un rapport actif de l'apprenant au savoir mathématique, a mis en évidence des normes telles que: être capable d'expliquer son interprétation et ses solutions, essayer de donner du sens aux explications des camarades ou encore questionner les alternatives. Les obser- 
vations empiriques ont montré que les normes de la microculture se constituent au fur et à mesure des contributions apportées par les membres de la communauté classe. Les normes ne sont donc pas imposées par l'enseignant, mais elles se négocient avec les élèves et sont amenées à évoluer au sein des interactions sociales. Elles sont considérées comme socialement reconnues et partagées par les membres de la classe, bien que ce partage ne soit a priori pas assuré. ${ }^{3}$ Il est en effet possible d'observer sur le plan individuel des conduites qui different des normes pourtant bien établies. De plus, comme le souligne Erickson (1986) dans une perspective interprétative de l'activité humaine, l'influence de la microculture sur les individus est un processus dynamique; l'interprétation individuelle des normes de la microculture peut varier selon les circonstances sociales, les exigences du contexte, la confrontation des perspectives individuelles au cours des processus participatifs aux pratiques sociales par exemple.

\section{Buts de la recherche}

Le but de l'étude est de tenter d'interroger les normes et les structures de participation telles qu'elles peuvent se manifester dans des activités mathématiques: Quels sont les systèmes d'attentes et d'obligations qui structurent les processus de participation des membres de la classe au cours de la résolution d'un problème mathématique? Dans quelle mesure ces attentes et obligations sont-elles représentatives de normes socialement reconnues et partagées par les membres de la communauté classe?

Létude détaillée de la leçon observée permet d'interroger les dynamiques interactives telles qu'elles se sont développées dans le cadre d'organisations sociales différentes, entre des temps de travail dyadique et une interaction semi-collective menée par l'enseignant. A partir de l'examen des processus interpersonnels, l'étude tente, d'une part, d'inférer quelques normes communautaires et, d'autre part, d'observer comment celles-ci s'actualisent au cours de l'interprétation et raisonnement mathématiques co-constitués au sein des processus participatifs.

\section{Méthodologie}

\section{Une étude de cas}

La leçon observée est traitée comme une étude de cas qui vise à décrire une situation spécifique, complexe et en évolution, dans un contexte déterminé (Stake, 1995). Si dans le cadre de cet article l'étude porte sur une seule leçon, à terme le but est d'observer plusieurs séries de leçons se déroulant dans deux classes, afin d'appréhender certains aspects contextualisés des processus d'enseignement et d'apprentissage dans une microculture de classe (Mottier Lopez, 2001). L'étude de cas ne portera plus sur l'unité «leçon», mais sur l'unité «communauté classe». 


\section{Contexte de la recherche et participants}

La recherche s'est déroulée dans le contexte d'une classe de quatrième année primaire, dans un établissement scolaire de Suisse romande implanté dans une zone géographique dont le milieu socio-économique est généralement moyen à supérieur. L'enseignant a un rapport positif aux moyens d'enseignement romands des mathématiques qu'il pratique depuis quatre ans. Il a organisé librement sa leçon.

Deux dyades sont plus particulièrement observées au cours des travaux de groupes. Les critères de choix des élèves sont fondés, d'une part, sur leur mode contrasté de participation aux interactions collectives observé au cours de séances précédentes. D'autre part, les élèves ont été sélectionnés en fonction de leur niveau scolaire en mathématiques qualifié sur la base de l'évaluation de l'enseignant.

\begin{tabular}{|c|}
\hline $\begin{array}{c}\text { Dyades observées } \\
\text { (prénoms modifiés) }\end{array}$ \\
\hline Sylvie (niveau scolaire élevé) \\
Anna (niveau scolaire faible) \\
Jean (niveau scolaire faible) \\
Béatrice (niveau scolaire moyen à faible) \\
\hline
\end{tabular}

Figure 1: Elèves observés

L'interaction semi-collective concerne, quant à elle, huit élèves - dont les deux dyades observées - et l'enseignant.

\section{Le corpus de données et L'analyse}

L'ensemble des interactions entre l'enseignant et les élèves au cours de la leçon, ainsi que les interactions entre les élèves pendant les phases de travail en dyades ont été enregistrées puis protocolées. Les traces écrites de la résolution du problème de chaque élève et des notes de terrain complètent le corpus de données.

A l'aide d'un instrument ad hoc, une analyse sur le plan mathématique - s'appuyant sur une analyse a priori du problème - et sur le plan social a été réalisée. Dans une approche de recherche qualitative et interprétative (e.g., Cobb, Stefan, McClain \& Gravemeijer, 2001; Erickson, 1986; Voigt, 1985), des hypothèses interprétatives sur les attentes et les obligations des membres de la communauté ont été faites en observant plus particulièrement les régularités des patterns d'interaction lorsque les individus tentent de coordonner leurs activités mathématiques.

Dans le cadre plus spécifique des interactions dyadiques, les résultats de recherche de Cobb (1995) montrent une stabilité des rôles et des systèmes d'attentes et d'obligations construits par les élèves au sujet de leurs propres activités et des activités des camarades dans une tâche mathématique. Mais pour les buts de notre propre recherche, il est apparu nécessaire de qualifier également les dynamiques sociales résultant de la construction interactive entre les élèves de leur 
relation interpersonnelle, afin de pouvoir observer, puis décrire, comment une même norme s'actualise au cours du développement de l'interprétation et du raisonnement mathématiques d'élèves de dyades différentes. C'est pourquoi un emprunt théorique a été fait en utilisant la catégorisation des formes d'interactions de co-élaboration entre pairs, définie par Gilly, Fraisse et Roux (1988). La première forme dite de co-élaboration acquiesçante désigne une dynamique interactive dans laquelle un des deux sujets élabore seul une proposition et la soumet à son partenaire. Ce dernier accepte la proposition en manifestant des feedbacks traduisant un véritable accord cognitif. La deuxième forme de co-élaboration désigne une co-construction entre les sujets sans expressions observables de désaccords ou de contradictions. La solution est conjointement élaborée avec des contributions des deux partenaires. Dans la troisième forme de co-élaboration, il s'agit de confrontations avec désaccords mais sans que les sujets n'argumentent les raisons de leurs désaccords ou formulent une nouvelle proposition. Enfin la quatrième forme de co-élaboration désigne des confrontations contradictoires entre les partenaires. Un sujet s'oppose à une proposition et argumente son désaccord ou propose une nouvelle procédure ou solution. Ce désaccord argumenté déclenche une phase de confrontation entre les partenaires afin de dépasser l'opposition (Gilly, Fraisse \& Roux, 1988, pp. 84-85).

Finalement, concernant les interactions semi-collectives, une première étape a consisté à identifier le type d'activité conjointe développée entre l'enseignant et les élèves, pour ensuite inférer les structures de participation en observant notamment l'exploitation collective des propositions mathématiques formulées par les élèves. Deux questions principales ont sous-tendu cette observation: comment l'enseignant reçoit-il la proposition de l'élève et pour quelle exploitation? quelle est la part prise par les autres élèves dans l'exploitation collective des propositions individuelles?

A des fins de validité, deux entretiens concernant la leçon observée ont eu lieu avec l'enseignant. Le premier entretien, le jour même de la leçon, a servi essentiellement à recueillir son point de vue sur les faits observés et sur les premières hypothèses interprétatives du chercheur. Le deuxième entretien, trois mois plus tard, a permis une restitution des interprétations et constats en vue d'une validité de signifiance (Pourtois \& Desmet, 1997).

\section{L'activité mathématique}

Il s'agit d'un problème, Une pomme pour la récré, qui s'inscrit dans le champ Reconnaître des problèmes multiplicatifs et divisifs. Le livre du maître stipule que la tâche consiste à résoudre une situation de partage comportant des opérations successives selon des règles plus ou moins complexes (Danalet et al., 1998). Le problème est formulé en ces termes aux élèves:

Une classe a reçu un carton plein de pommes. Quel jour de la semaine le carton sera-t-il vide?

On dispose des renseignements suivants: 
- le carton contient 185 pommes

- il y a 22 élèves dans la classe

- la distribution des pommes commence un lundi

- chaque élève reçoit une demi-pomme par jour

- il y a 5 jours d'école par semaine

Sur la base de la typologie des classes de problèmes définies par Vergnaud (1981, 1988), une analyse a priori de l'activité a été faite. Il ressort que quatre "grandeurs» sont en relation plus ou moins directe les une avec les autres dans le problème: des quantités de pommes, d'élèves, de jours et de semaines. Les relations relèvent toutes de la proportionnalité. On se trouve donc en présence de quatre suites proportionnelles, chacune dans son "espace de mesures». Dans cet article, seule la relation principale en jeu est plus particulièrement présentée. ${ }^{4}$ Celle-ci implique deux espaces de mesures: le nombre de jours et le nombre de pommes. Le tableau de correspondance suivant illustre cette relation:

\begin{tabular}{c|c}
$\begin{array}{c}\text { Nb de } \\
\text { Jours }\end{array}$ & $\begin{array}{c}\text { Nb de } \\
\text { pommes }\end{array}$ \\
\hline 1 & $1 / 2$ \\
$?$ & 185
\end{tabular}

La première étape consiste à déterminer le nombre de pommes correspondant à un jour ${ }^{5}$, en interprétant les données 22 élèves dans la classe; une demi-pomme distribuée par jour à chaque élève. Cette interprétation demande de considérer la mesure «nombre d'élèves» mise en relation avec la mesure «nombre de pommes», afin de trouver la quantité de pommes distribuées pour un jour aux 22 élèves.

\begin{tabular}{c|c}
$\begin{array}{c}\text { Nb de } \\
\text { d'élèves }\end{array}$ & $\begin{array}{c}\text { Nb de } \\
\text { pommes }\end{array}$ \\
\hline 1 & - \\
22 & $?$
\end{tabular}

Une fois celle-ci trouvée, on peut retourner au tableau de correspondance de la relation principale du problème, complété par la valeur unitaire 11. Ensuite, les élèves ont la possibilité d'appliquer deux types de calcul relationnel, soit par isomorphisme de mesures - une relation centrée sur la notion d'opérateur «scalaire» - soit centrée sur la relation fonctionnelle.

Rappelons que la procédure scalaire est obtenue par une augmentation proportionnelle des deux mesures impliquées dans la relation, autrement dit, on assiste à une augmentation parallèle des mesures. Dans Pommes pour la récré, cela signifie que si, au cours d'un jour, 11 pommes sont mangées, au cours de deux jours, deux fois plus de pommes seront mangées. Dans la même logique, pour 10 jours, cela représentera 10 fois la quantité de pommes mangées pour un jour. 


\begin{tabular}{|c|c|c|}
\hline \multicolumn{2}{|c|}{$\begin{array}{c}\mathrm{Nb} \text { de } \\
\text { Jours }\end{array}$} & $\begin{array}{c}\mathrm{Nb} \text { de } \\
\text { pommes }\end{array}$ \\
\hline 1 & \multirow[b]{3}{*}{$x$ ? } & 11 \\
\hline 2 & & 22 \\
\hline$\alpha$ & & \\
\hline 10 & & 110 \\
\hline
\end{tabular}

Quant à la procédure fonctionnelle, elle présente l'une des deux mesures du problème comme une fonction de l'autre mesure. Par exemple, le nombre total de pommes est fonction du nombre de pommes correspondant à un jour. Le facteur fonction 11 est constant pour toutes les quantités de pommes. Cependant, comme le souligne Nunès (1991), «les procédures fonctionnelles sont plus éloignées du sens que les solutions scalaires parce qu'elles impliquent le calcul croisé des variables» (p. 125).

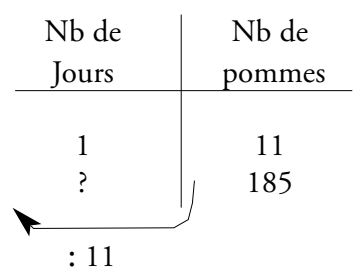

Une des difficultés du problème est que les élèves vont devoir considérer la notion de reste lors de l'interprétation des opérations mathématiques formulées, afin de pouvoir répondre à la question du problème. D'une part, ils devront interpréter les 16 jours de distribution en terme de semaines de cinq jours. Autrement dit, il reste un jour qui est le lundi de la 4ème semaine en considérant que la distribution des pommes commence un lundi. D'autre part, les élèves devront également considérer le reste de la division euclidienne "188: 11» qui stipule qu'il y a neuf dernières pommes à distribuer le mardi de la quatrième semaine, tout en précisant que ce jour-là, quatre élèves ne recevront pas de pommes. Bien que l'intention didactique du problème soit le partage de collections, on peut s'attendre à des procédures diverses, faisant intervenir des suites d'additions réitérées, des combinaisons d'additions, des combinaisons de multiplications et d'additions, des suites de soustractions successives.

L'objectif de cette analyse a priori est de fournir un cadre d'analyse au raisonnement mathématique des élèves tel qu'il s'est effectivement développé au sein des différentes organisations sociales de la leçon et vu comme se constituant dans les discours interactifs des participants (Resnick, Pontecorvo \& Säljo, 1997). En ce faisant, il est tenté d'appréhender les systèmes d'attentes et d'obligations qui structurent les interactions de co-élaboration de ce raisonnement, compte tenu que ces systèmes se manifestent non seulement de façon implicite, mais qu'ils 
sont une partie intégrale de l'activité des élèves selon l'hypothèse théorique des perspectives situées.

\section{Résultats}

\section{Déroulement de la leçon}

L'enseignant a choisi de partager la classe en trois groupes de base de huit élèves qui réalisent une activité mathématique différente sur le temps d'une leçon d'environ 50 minutes. Un «tournus» est organisé sur plusieurs séances. Le groupe de base concerné par l'activité Une pomme pour la récré est subdivisé en quatre dyades dont les interactions de deux dyades sont ici plus particulièrement étudiées. En cours de leçon, une mise en commun a réuni les quatre dyades seulement, d'où la qualification d'interactions semi-collectives de cette organisation sociale.

\begin{tabular}{|c|c|c|c|}
\hline $\begin{array}{c}\text { Organisation du travail } \\
\text { par l'enseignant }\end{array}$ & $\begin{array}{c}\text { Première phase de réso- } \\
\text { lution en dyades }\end{array}$ & $\begin{array}{c}\text { Mise en commun } \\
\text { semi-collective }\end{array}$ & $\begin{array}{c}\text { Reprise de l'activité en } \\
\text { dyades }\end{array}$ \\
\hline $\begin{array}{c}2 \text { minutes } \\
\text { (temps arrondi) }\end{array}$ & 16 minutes & 10 minutes & 26 minutes \\
\hline
\end{tabular}

Figure 2: Phases de déroulement de la leçon

\section{Première phase de résolution de problèmes de Sylvie et d'Anna}

Les interactions entre Sylvie et Anna (voir figure 3) se déroulent principalement sur un mode d'élaboration acquiesçante (Gilly, Fraisse \& Roux, 1988): Sylvie élabore la solution en proposant des opérations successives compte tenu de son interprétation et raisonnement mathématiques. Elle énonce dans un premier temps la division "185: 11», témoignant qu'elle a déjà effectué mentalement soit la division «22: 2 », soit la multiplication lacunaire « $2 \times \ldots=22 »$, permettant de connaître la valeur unitaire de la relation «nombre de jours» - «nombre de pommes». Elle poursuit en suggérant de rechercher le nombre de pommes mangées dans une semaine de cinq jours, pour ensuite développer une résolution par additions successives des quantités de pommes «semaines», «jours» et «reste». Anna accepte et suit les propositions de sa camarade. Sa contribution à la résolution se situe notamment en énonçant les résultats intermédiaires et en reformulant les propositions de Sylvie.

Bien que la situation de partage soit initialement identifiée avec l'énonciation de la division euclidienne "185: 11», les élèves, qui ne connaissent par encore l'algorithme de cette opération, choisissent une résolution par additions successives des quantités de pommes «semaine», «jour» et «reste». En ce sens, les élèves 
développent une procédure scalaire, alors qu'elles réfléchissent en terme de «nombre de fois» d'une quantité de pommes définie. Le dénombrement de chaque quantité permet ensuite d'interpréter le nombre de jours et de semaines pour répondre à la question du problème. On note que la relation entre les mesures «nombre de jours et semaines» et "quantité de pommes distribuées» reste à l'esprit de Sylvie qui ajuste la valeur des nombres à additionner pour atteindre 185 au fur et à mesure de la résolution. Anna, quant à elle, semble parfois éprouver des difficultés à établir des liens entre les calculs effectués sous la guidance de Sylvie et les données du problème.

\begin{tabular}{|c|c|c|}
\hline $\begin{array}{l}22: 2=11 \\
2 \times 11=22 \\
185: 11\end{array}$ & $\begin{array}{l}\text { Identification de la valeur unitaire } \\
\text { de la relation "nb de jours " "nb } \\
\text { de pommes "et énonciation de la } \\
\text { division euclidienne « } 185: 11 \text { " }\end{array}$ & \multirow{2}{*}{$\begin{array}{l}\text { Calcul de la valeur " quantité } \\
\text { pommes/jours " et de la valeur " } \\
\text { quantité pommes/semaine". }\end{array}$} \\
\hline $11 \times 5=55$ & $\begin{array}{l}\text { Calcul de nombre de pommes } \\
\text { consommées par semaine ; ou cal- } \\
\text { cul d'un «paquet » qui permet } \\
\text { d'arriver plus rapidement à la solu- } \\
\text { tion. }\end{array}$ & \\
\hline $\begin{array}{l}2 \times 55=110 \\
110+55=3 \times 55=165\end{array}$ & $\begin{array}{l}\text { Additions réitérées; multiplication } \\
\text { de la quantité pommes/semaine. }\end{array}$ & \multirow{3}{*}{$\begin{array}{l}\text { Additions successives de différentes } \\
\text { quantités de pommes pour at- } \\
\text { teindre } 185 \text { et interprétation des } \\
\text { quantités en terme de nombre de } \\
\text { semaines et jours. }\end{array}$} \\
\hline $165+11=176$ & $\begin{array}{l}\text { Addition de la quantité } \\
\text { pommes/jour }\end{array}$ & \\
\hline $176+9=185$ & $\begin{array}{l}\text { Addition du reste, quantité } \\
\text { pommes < quantité pommes/jour }\end{array}$ & \\
\hline
\end{tabular}

Figure 3: Procédure de résolution de Sylvie et d'Anna

La relation entre les élèves est asymétrique: Sylvie apparaît comme l'autorité mathématique du groupe, une autorité non remise en question par Anna. L'inférence des attentes et obligations que chaque élève semble avoir par rapport à son activité et à celle de son camarade permet de caractériser cette relation asymétrique, dans le sens qu'on observe que tout au long de la résolution, Sylvie explique fréquemment son raisonnement à Anna. Parfois même, elle joue «au petit maître» en lui posant des questions auxquelles Anna répond avec complaisance. Quant à Anna, elle participe en reformulant sa compréhension des propositions faites par Sylvie. Elle n'hésite pas à poser des questions lorsqu'elle ne suit pas le raisonnement de sa camarade.

Sylvie

Ça fait déjà trois semaines.

Anna

Trois semaines?

Sylvie

Oui trois semaines. Cinquante-cinq c'est si on mange des pommes en une semaine. La classe consomme des pommes en une semaine, alors après fois trois, ça fait trois semaines. [...] 
Anna Donc il faut arriver à 185 (3 sec.) Ben maintenant il faut viser plus haut. Par exemple euh.

Sylvie Mais si tu rajoutes 55 ça fera trop gros.

Anna $\quad M m$.

Sylvie Alors il faut rajouter jour par jour pour calculer.

Anna Donc tu veux dire que si on fait quatre fois 55 ce serait déjà trop?

Sylvie Ouais il n'y aurait pas assez de pommes. (3 sec.) Pas assez de pommes. (3 sec.)

Anna Bon alors on pourrait faire 155 aller à 185?

Sylvie Ouais, mais il faut rajouter toujours euh rajouter un jour et puis un jour d'accord? Parce qu'on peut pas prendre des pommes comme ça au hasard.

D’une façon générale, on observe que Sylvie se sent obligée 6 d'expliquer son raisonnement à Anna, qui, quant à elle, essaie de donner sens aux explications de Sylvie. Cette structure d'interaction sous-tend l'ensemble de l'activité de résolution de problèmes des deux élèves.

\section{Première phase de résolution de problèmes de Béatrice et Jean}

Contrairement à la dyade de Sylvie et Anna qui a fonctionné sur un type relativement stable de dynamique interactive, le mode d'interaction entre Jean et Béatrice est plus variable. Au début de la résolution, les élèves construisent conjointement l'interprétation du problème et semblent partager la même approche du problème:

Jean Alors 185 pommes ( 3 sec.) Tu vois, ils sont 22 élèves donc dans une classe. Alors il faut faire euh

Béatrice Combien de pommes. Il y a 22 élèves. Donc euh

Jean 185 pommes et puis il y a cinq jours d'école par semaine. Ça veut dire que cinq jours, ils reçoivent toujours une pomme d'un jour à l'autre si je ne me trompe pas.

Béatrice Ouais.

Jean On fait combien, on va faire combien pour aller jusqu'à 22. Euh! Pour aller jusqu'à 185 plutôt.

Béatrice Combien de fois il y a 22 dans

Jean Dans 185.

Béatrice Ouais dans 185.

Jean Tu divises 22 plutôt [...] parce que chaque élève reçoit une demipomme par jour.

Béatrice Ouais alors attends. Ben tu fais 11, 11 pommes, 11 pommes.

Jean Parce que si tu partages par la moitié, ben tu donnes à un élève et puis à un autre. Après on reprend une pomme et puis on la partage par la moitié. C'est comme si c'était la pomme entière. 
Les élèves semblent initialement s'accorder sur le fait qu'il faut diviser 22 par 11, compte tenu que les élèves mangent une demi-pomme par jour. Toutefois, l'ensemble des interactions suivantes va porter sur cette assertion qui sera finalement réfutée et qui donnera lieu à des interprétations et raisonnements différents entre les élèves. En effet, Jean paraît se centrer sur la quantité d'élèves dans la classe vue comme la valeur à réitérer pour atteindre 185 . Toujours dans une dynamique de co-construction, les élèves vont chercher à prendre en compte l'interprétation du camarade en tentant d'élaborer conjointement une nouvelle opération. Cela amène Béatrice à confondre une «demi-pomme» et une "pomme et demi». De son côté, Jean tente aussi de proposer une suite de résolution fondée sur les propositions de Béatrice. Cependant, au fil des interactions et compte tenu des divergences d'interprétation de plus en plus marquées entre les élèves, quelques interactions de confrontation avec ou sans essais d'argumentation ont lieu. Ces confrontations ne sont pas couronnées de succès. En effet, malgré les efforts des deux élèves pour élaborer une solution conjointe, Béatrice et Jean ne parviennent pas à développer une base commune de compréhension et de communication, notamment concernant l'interprétation des données relatives à la demi-pomme distribuée à chaque élève. Dans la mesure où cette opération est fondamentale pour la résolution du problème et que les élèves ne parviennent pas à surmonter cette difficulté, Jean et Béatrice paraissent peu avancés dans la résolution au début de la mise en commun, comparativement aux trois autres dyades.

\begin{tabular}{|c|c|c|}
\hline$? \times 22=185$ & \multicolumn{2}{|l|}{ Accord entre les élèves } \\
\hline $22: 2=11$ & \multicolumn{2}{|c|}{ Accord initial, puis désaccord entre les élèves } \\
\hline $\begin{array}{l}\text { Jean } \\
? \times 22=185\end{array}$ & $\begin{array}{l}\text { Béatrice : confusion entre } 0,5 \\
\text { pomme et } 1,5 \text { pomme } \\
1,5+1,5+1,5+\ldots=185 \quad \text { ? } \\
\times 1,5=185 \\
\text { Nb de pommes } \\
3 \\
3 \\
3 \\
\ldots 185 \\
\text { Nb d'élèves } \\
2 \\
2 \\
2 \\
\ldots\end{array}$ & $\begin{array}{l}\text { Les élèves ne parviennent pas à } \\
\text { comprendre leur raisonnement } \\
\text { respectif. }\end{array}$ \\
\hline
\end{tabular}

Figure 4: Début de la résolution de Béatrice et Jean

Les attentes et obligations inférées au fil de ces interactions montrent que Béatrice et Jean cherchent tous deux à développer une résolution conjointe du problème, ce qui les amène à prendre en compte l'avis du camarade et à explorer plu- 
sieurs pistes. Au vu de leurs divergences d'interprétation, ils auraient pu choisir, par exemple, de poursuivre la résolution du problème de façon plus individuelle. Ce n'est pas le choix des enfants. Lorsque Béatrice émet une proposition, elle explique son raisonnement et l'opération projetée à Jean avant même de l'écrire et de l'effectuer. Jean tente également d'expliquer son interprétation et son raisonnement à Béatrice, bien qu'il paraisse moins à l'aise.

\section{Phase de mise en commun}

L'analyse du déroulement de la mise en commun qui, rappelons-le, réunit quatre dyades est fondée sur l'identification des activités conjointes développées entre l'enseignant et les élèves, permettant ainsi de délimiter des phases successives.

\begin{tabular}{|l|l|l|}
\hline TdP & Phases & Paroles «signal» de l'enseignant \\
\hline $1-26$ & $\begin{array}{l}\text { Enumération des variables de } \\
\text { l'énoncé du problème }\end{array}$ & $\begin{array}{l}\text { Qu'est-ce que vous pouvez me dire sur les consignes? Qu'est- } \\
\text { ce qui est écrit dans le problème? Qu'est-ce que vous avez re- } \\
\text { marqué? }\end{array}$ \\
\hline $27-85$ & $\begin{array}{l}\text { Explication des différentes pro- } \\
\text { cédures de résolution projetées } \\
\text { par les dyades }\end{array}$ & $\begin{array}{l}\text { Alors j'aimerais que vous puissiez expliquer en deux mots } \\
\text { comment vous allez vous y prendre pour résoudre ce pro- } \\
\text { blème? Vous n'avez pas nécessairement tous la même solu- } \\
\text { tion. }\end{array}$ \\
\hline $85-103$ & $\begin{array}{l}\text { Résumé des propositions des } \\
\text { élèves }\end{array}$ & $\begin{array}{l}\text { Alors si on résume les différentes manières que vous avez de } \\
\text { faire... }\end{array}$ \\
\hline
\end{tabular}

\section{Figure 5: Déroulement de la mise en commun}

Un premier constat est que la dynamique interactive n'est pas identique dans les trois phases constitutives de la mise en commun.

Dans la première phase, la consigne formulée par l'enseignant amène les élèves à énumérer les variables du problème. Après chaque énonciation d'une donnée pertinente, l'enseignant valide la proposition de l'élève. En ce sens, cette première phase sert essentiellement à identifier collectivement les données de l'énoncé du problème, sans intention de discussion ni de débat particulier.

La deuxième phase est introduite par la consigne suivante: blème.

- Expliquer en deux mots comment vous allez vous y prendre pour résoudre ce pro-

Cette requête n’apparaît plus par la suite. Spontanément les élèves expliquent l'interprétation et le raisonnement mathématiques développés dans leur dyade. Ce constat permet de postuler que les élèves sont habitués à expliciter leur procédure de résolution dans la mise en commun.

Le premier élève à prendre la parole est Jean qui tente d'expliquer son raisonnement en énonçant à la fois des éléments de sa propre interprétation et de celle de sa camarade. 
Jean

On sait déjà que dans le carton il y a 185 pommes, et puis qu'ily a 22 élèves, et puis qu'ils reçoivent une demi-pomme par jour. [...] Ben quand ils reçoivent une demi-pomme, elle partage et puis elle en donne un à un élève et puis à un autre. Comme si la pomme était entière. Ben après on peut faire plus trois.

Enseignant Alors qu'est-ce que vous en pensez les autres? (3 sec)

Thierry Ben jai pas tout compris.

Jean C'est comme si t'as, par exemple, t'as le maître qui prend la pomme. Ben il la partage et après il donne a chacun à deux élèves. Si t'avais deux pommes, si tu partageais la pomme comme ça, ben ça peut faire trois.

Thierry Ça fait pas trois, ça fait quatre!

Béatrice n'interviendra à aucun moment de la mise en commun pour rendre public son interprétation ou pour soutenir les propos de Jean. Au vu de sa forte implication dans l'interaction dyadique, cette attitude réservée dans l'interaction collective - attitude qualifiée d'habituelle par l'enseignant - met en évidence les modes contrastés de participation d'un même élève dans différentes organisations sociales de la leçon.

Sylvie est la suivante à expliquer son raisonnement. Elle le fait en un seul tour de parole:

Sylvie

Ça fait la moitié de 22, parce que chaque élève ne reçoit pas une pomme entière. Et puis ensuite ça fait 11, la moitié de 22. Et puis combien de fois il y a dans une semaine. 11 fois cinq.

Enseignant Bien, OK. Et toi André?

Tout comme Béatrice, Anna n'interviendra jamais pour expliquer le travail de son groupe.

Les élèves des deux dernières dyades s'expriment à leur tour. Ils expliquent des procédures non abouties, qui toutes deux ne demandent pas de diviser 22 par deux. En effet, les élèves choisissent de raisonner en considérant 22 fois une demi-pomme, ce qui implique de doubler le nombre total de pommes.

Après l'explication fournie par l'élève, à l'exception de la proposition de Sylvie, l'enseignant ne valide pas la pertinence de l'interprétation et du raisonnement exposés. Il répond par une question qui sollicite l'avis des autres élèves:

- Alors qu'est-ce que vous en pensez les autres?

- Est-ce que vous êtes d'accord avec ça?

- Vous avez compris les autres?

Ces questions incitent les élèves à donner du sens aux propositions de leurs camarades, et à manifester si nécessaire leur incompréhension. Ainsi, Thierry dit à Jean qu'il n'a pas compris son raisonnement. De même, Jean et Béatrice expriment leur incompréhension relativement aux procédures qui proposent de raisonner avec la valeur unitaire 22 plutôt que 11 et de doubler le nombre total de pommes. En réponse aux manifestations d'incompréhension, on note que l'enseignant ne reformule pas la proposition initialement énoncée, mais il sollicite 
une nouvelle explication de l'auteur de la proposition. Cela fournit des occasions de discussion entre les élèves qui ne comprennent pas le raisonnement mathématique exposé ou qui ne sont pas d'accord avec les propositions de leurs camarades.

On relève que la mise en commun ne sert pas à énoncer la suite d'opérations mathématiques projetées ou déjà réalisées par les dyades. Par exemple, bien qu'étant informé que le groupe de Sylvie et Anna ait déjà trouvé la réponse à la question du problème, l'enseignant ne sollicite par cette réponse, ni même une description détaillée des opérations successives amenant à l'obtention du résultat. Sylvie et Anna ne cherchent pas non plus à communiquer leur procédure et résultat, conscientes peut-être que ce n'est pas l'attente de l'enseignant dans cette mise en commun.

L'enseignant précise encore qu'il n'attend pas une seule procédure de résolution possible:

- Vous n'avez pas nécessairement tous la même solution.

Il accepte la diversité des procédures et il n'émet pas de commentaires évaluatifs. En ce sens, les élèves ne devraient pas chercher à trouver «la» procédure attendue par le maître, mais ils sont incités à développer leur propre démarche de résolution. Par contre, une des contraintes relatives à cette interaction semi-collective est d'être capable d'expliquer aux autres membres de la classe l'interprétation et le raisonnement mathématiques développés. Par la sollicitation de manifestations d'accord ou de désaccord, de compréhension ou d'incompréhension, les élèves sont amenés, d'une part à tenter de donner sens aux propositions des camarades et, d'autre part, à discuter les propositions.

La dernière phase de la mise en commun sert à la reformulation par l'enseignant des différentes procédures mises en présence. Tout comme dans la première phase, un mode plus traditionnel d'interaction entre l'enseignant et les élèves a lieu, fondé notamment sur le pattern d'interaction: question fermée de l'enseignant, réponse de l'élève, feedback de l'enseignant sous forme de validation de la pertinence de la réponse de l'élève. Ces interactions permettent d'institutionnaliser la valeur unitaire de la relation multiplicative «nombre de jours» «nombre de pommes», une donnée essentielle pour rechercher ensuite la quantité d'unités «jours» pour 185 pommes. On observe que l'enseignant accepte les différentes interprétations: 11 si l'on raisonne en terme de pommes entières et 22 si l'on raisonne en nombre de demi-pommes, mais qui implique que le nombre total de pommes soit doublé.

Globalement, on constate que selon le type d'activité conjointe, la dynamique interactive entre l'enseignant et les élèves est différente. Relativement traditionnelle lorsqu'il s'agit d'identifier les données présentes dans l'énoncé du problème, ainsi que d'institutionnaliser la valeur unitaire de la relation multiplicative, la dynamique de l'interaction collective donne au contraire une large place à des patterns d'explicitation et de discussion de l'interprétation et du raisonnement mathématiques sous-jacents à la procédure de résolution projetée ou déjà développée dans chaque dyade. 
Phase de reprise de la résolution de problèmes de Sylvie et Anna

Alors que Sylvie et Anna ont trouvé la solution du problème, l'enseignant leur demande de reprendre le travail avec la consigne qu'Anna soit capable d'expliquer à Sylvie l'entier de la procédure déployée.

Anna Alors d'abord on prend eub cinq jours parce que chaque semaine il y a cinq jours d'école.

Sylvie Oui.

Anna Et puis, comme il y a 22 élèves on doit diviser 22 élèves par deux.

Sylvie Pourquoi on doit diviser par deux?

Anna Ben parce qu'il y a chaque élève qui reçoit une demi-pomme par jour, donc on a divisé 22 par 11 et puis après on met, on a essayé de faire combien de fois. Ah non! D'abord on fait cinq fois 11. Ça fait 55 et puis après on fait

Sylvie Mais pourquoi on a fait cinq fois 11 ?

Anna Ben parce qu'il y a cinq jours dans la semaine

Sylvie Ouais

Il est intéressant de noter ici l'inversion des rôles, alors que dans la phase initiale de recherche, c'est essentiellement Sylvie qui, au fil de la résolution du problème, a expliqué la démarche à Anna. Globalement, on constate que Sylvie joue quasiment le rôle de l'enseignant qui pose des questions et valide les réponses. Anna ne s'en offusque pas, un signe peut-être que cela correspond à l'attente qu' elle a du rôle de Sylvie.

Phase de reprise de la résolution de problèmes de Béatrice et Jean

Tout comme dans la première phase de la leçon, la forme de dynamique interactive développée entre les élèves varie. Béatrice et Jean cherchent à obtenir une solution commune, alors qu'ils choisissent une résolution par additions successives de la valeur 11, choix qui peut être vu comme un effet de régulation des interactions semi-collectives de la mise en commun (Allal, 1988; Mottier Lopez, 1999), Béatrice propose la multiplication «11 x 11». Des désaccords plus ou moins argumentés ont lieu à propos du produit de celle-ci. Finalement les élèves s'accordent sur 121. Ensuite, Béatrice prend l'initiative de la résolution. Elle formule des propositions pour interpréter « $11 \times 11$ » en terme de nombre de semaines et de jours. Elle se sent toutefois obligée de demander à son camarade s'il comprend son raisonnement. Jean qui semble refuser le rôle un peu passif que tout à coup lui attribue Béatrice, profite de formuler une objection argumentée. Les échanges suivants se poursuivent sur un mode de co-construction avec le calcul des sommes intermédiaires jusqu’à 165 .

Soudainement, Béatrice prend conscience qu'ils ne parviendront pas à la somme de 187 en réitérant la valeur 11. Les deux élèves pensent que c'est le produit de «11 x 11» qui est incorrect et ils décident de contrôler cette multiplica- 
tion qui initialement avait causé des difficultés. Des désaccords ont à nouveau lieu. Chacun essaie d'argumenter son point de vue. Mais Béatrice se fâche. Jean tempère. Les deux élèves reprennent la résolution sur un mode de co-construction à partir d'une réponse intermédiaire consensuelle. Finalement, Jean constate que la réponse 121 est correcte et que par conséquent ils n'atteindront pas 185 avec la procédure mathématique envisagée. Les élèves commencent à s’impatienter:

Jean

Moi je te dis que ça va pas marcher.

Béatrice Et ben, on fait plusieurs calculs. Comme ça, on est un peu sûrs. Alors, maintenant tu fais le calcul sur ta feuille, au lieu que ce soit tout moi qui dois faire sur ma feuille.

Jean Mais c'est pas ça Béatrice! C'est que ça va pas marcher, parce que regarde si on fait plus 11 [...] parce qu'après si tu fais $99+11$, ça va faire 110. Et puis $11 \times 11$ ça va faire 121. C'est comme si c'est $110+11$ (3 sec) $110+11$ ça va faire 121 . Tu peux pas aller jusqu'à 185 .

L'enseignant intervient et valide la pertinence des sommes intermédiaires trouvées. Les élèves reprennent sur un mode de co-construction, avec des conduites d'explication mutuelle, mais ils ne parviennent pas à concevoir une interprétation permettant de répondre à la question du problème.

\begin{tabular}{|c|c|}
\hline $11 \times 11=121$ & 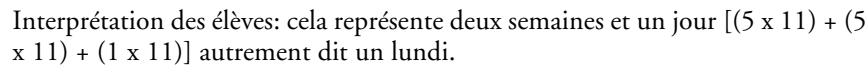 \\
\hline $121+11=132$ & \multirow{6}{*}{$\begin{array}{l}\text { Addition successive de la valeur d'un jour de distribution de pommes. } \\
\text { Les élèves n'interprètent pas ces additions en terme de jours supplémentaires. Ils } \\
\text { sont bloqués par le fait que } 185 \text { n'est pas un multiple de } 11 \text {; la notion de reste } \\
\text { fait obstacle. }\end{array}$} \\
\hline $132+11=143$ & \\
\hline $143+11=154$ & \\
\hline $154+11=165$ & \\
\hline $165+11=176$ & \\
\hline $176+11=187$ & \\
\hline
\end{tabular}

Figure 6: Procédure de résolution de Jean et de Béatrice

Sur le plan mathématique, on observe que Béatrice et Jean développent une procédure de résolution scalaire, en raisonnant d'abord dans un espace de mesures pour ensuite interpréter les opérations dans l'autre espace de mesures. Mais la notion de reste fait obstacle, autrement dit les élèves n'envisagent pas qu'il puisse rester une quantité de pommes plus petite qu'un jour de distribution. Cela les empêche de poursuivre leur raisonnement relativement à la mesure «nombre de jours», un raisonnement qui avait été entrepris pour l'opération «11 x 11 ». D’une façon générale, il semble que Jean éprouve des difficultés à identifier les mesures pertinentes de la relation multiplicative de la situation proposée. Il est 
par contre très actif lorsqu'il s'agit de formuler des opérations et leurs résultats. Globalement, chez les deux élèves, la résolution du problème s'appuie sur une conception essentiellement additive. La multiplication sert surtout à compter le nombre d'itérations.

Sur le plan social, on constate que les élèves considèrent que la résolution du problème doit se faire conjointement, mais ils semblent éprouver quelques difficultés à gérer les désaccords qui surviennent. Certaines prises de parole de Béatrice laissent entendre qu'elle ait été tentée d'imposer son interprétation et raisonnement à Jean. Celui n'accepte pas et continue d'attendre de la part de sa camarade qu'elle prenne en compte son point de vue et ses suggestions. Béatrice accepte cette attente de Jean en recommençant à considérer l'avis de son camarade. Ce constat est révélateur des processus, souvent implicites, de négociation des attentes et obligations mutuelles qui structurent les interactions et les activités entre les individus (Voigt, 1985).

\section{Synthèse et discussion}

Bien que des éléments de synthèse sur l'interprétation et le raisonnement mathématiques des élèves pourraient être discutés (Mottier Lopez, 2002), la synthèse porte ici essentiellement sur les résultats de l'analyse des structures de participation inférées à partir des processus interpersonnels entre les élèves et avec l'enseignant.

Un élément commun se dégage des différents processus interactifs analysés: s'expliquer et se comprendre mutuellement. Il ressort qu'une grande partie de l'interaction semi-collective sert à l'explication mutuelle de l'interprétation faite $\mathrm{du}$ problème. A une seule reprise, l'enseignant sollicite cette explication sous forme de consigne explicite. Ensuite, les élèves qui prennent la parole le font spontanément. On peut donc postuler que l'attente de l'enseignant est perçue comme une norme par les élèves. De même, l'enseignant incite les élèves à comprendre les propositions des camarades en sollicitant une manifestation d'accord ou de compréhension. Les élèves qui s'expriment le font sans difficultés apparentes. En ce sens, l'explication et la recherche de compréhension mutuelle, dans l'interaction collective, apparaissent comme socialement reconnus et partagés par la communauté classe. Mais cette structure d'interaction n'est pas seulement réservée aux interactions semi-collectives. Elle est également présente dans les interactions dyadiques et il devient intéressant d'observer comment elle s'actualise dans la dynamique sociale propre à chaque groupe.

Dans le cadre d'une dynamique interactive sous forme d'élaboration acquiesçante, Sylvie explique son raisonnement et les opérations mathématiques conçues au cours de la phase initiale de résolution de problèmes. Anna, sa partenaire, collabore dans le sens qu'elle cherche à comprendre Sylvie et, pour ce faire, elle sollicite des explications de sa camarade. Lors de la phase de reprise de l'acti- 
vité, après la mise en commun, Anna est amenée à son tour à développer des activités d'explication, mais, par contre, ayant trait à une procédure de résolution déjà élaborée. Sylvie la guide en posant des questions et en validant au fur et à mesure les explications de sa camarade.

Béatrice et Jean, fonctionnent, quant à eux, principalement sur un mode de co-construction, régulièrement ponctué de brefs échanges relevant de confrontations plus ou moins argumentées. Les deux élèves verbalisent leur interprétation et raisonnement tout au long de la résolution. Béatrice explique ses propositions soit spontanément ou à la demande de Jean qui, d'ailleurs, n'hésite pas à manifester son incompréhension. Bien que Béatrice paraisse solliciter moins volontiers les explications de son camarade, Jean cherche régulièrement à expliquer, lui aussi, son raisonnement. Globalement, les deux élèves semblent attentifs à établir une compréhension mutuelle, bien qu'ils éprouvent parfois des difficultés à construire précisément cette compréhension qui, dans les faits, n’est pas toujours partagée comme le montrent les analyses effectuées.

Ces constats mettent en évidence que l'explication et la recherche de compréhension mutuelle caractérisent les processus participatifs aux pratiques mathématiques de la classe, à la fois dans l'interaction semi-collective et lors des activités des deux dyades observées. Cependant il conviendrait de s'interroger sur la question de la variation qualitative d'une même structure de participation dans différentes organisations sociales et en fonction de la nature des activités conjointes. Les élèves expliquent-ils de la même manière dans un temps collectif, en présence de l'enseignant, et dans le cadre d'interactions entre pairs? De plus, dans quelle mesure ce système d'attentes et obligations dans l'interaction dyadique est-il spécifique à la dynamique relationnelle de quelques élèves en particulier ou est-il plus largement partagé par les membres de la classe? Un épisode à la fin de l'interaction semi-collective apporte quelques éclairages sur cette dernière question:

Enseignant Est-ce que tu as suivi ce qu’à dit Sébastien? (le partenaire d'André)

André Non je n'ai pas très bien compris.

Enseignant Alors quel est ton rôle, ton devoir?

André Ben euh, de dire qu'il réexplique.

Enseignant Eh oui. D'accord hein. C'est important pour que tu sois au clair avec ça. Parce que recopier les calculs, c'est pas très très utile. Tu dois pouvoir arriver à faire aussi. Hein Laurine? (la partenaire de Thierry)

Laurine Euh...

Enseignant Alors la même chose. Si c'est trop compliqué, la manière de faire de Thierry, ou bien tu redemandes une explication, ou bien tu fais quelque chose de plus simple.

Ces échanges montrent que l'explication et la compréhension mutuelle répondent à une attente enseignante relative au «rôle» et au «devoir» des élèves les uns 
par rapport aux autres dans les travaux de groupe. Suite au constat, dans l'interaction semi-collective, d'une absence supposée de cette structure de participation dans un groupe, l'enseignant reformule explicitement son attente. Il conviendrait d'ailleures de se demander ici s'il s'agit d'une absence de la structure de participation ou d'une absence de l'effet de cette structure. Il se pourrait, par exemple, que les élèves aient cherché à s'expliquer et se comprendre mutuellement au cours leur travail dyadique mais sans que cela produise l'effet escompté par l'enseignant.

Cet épisode interactif permet de postuler que l'explication et la compréhension mutuelle dans les travaux de groupes représentent, sur le plan communautaire, une norme reconnue et partagée de la microculture de classe (Yackel \& Cobb, 1996). 7 Dans la réponse d'André, sollicité par l'enseignant d'expliciter son rôle et son devoir dans les travaux de groupes, on note que l'élève est capable de nommer cette norme. En ce sens, une des spécificités de la norme inférée dans notre étude est d'avoir été explicitement constituée, alors que les observations empiriques de Voigt (1985, 1995) montrent que les normes s'établissent fréquemment de façon implicite au cours des interactions entre l'enseignant et les élèves. Ce constat incite à questionner plus en avant le jeu de l'implicite et de l'explicite dans la constitution d'une microculture de classe. De plus, on observe que la norme «s'expliquer et chercher à se comprendre mutuellement» tend à promouvoir une co-élaboration de la résolution entre les élèves, plutôt qu'une recherche individuelle de la solution du problème comme cela pourrait se produire dans des travaux de groupes qui ne comportent pas une structure collaborative contraignante.

\section{Brève conclusion}

Des théoriciens du paradigme des perspectives situées (Brown, Collins \& Duiguid, 1989; Collins, Brown \& Newman, 1989) développent le modèle d'apprentissage "cognitif apprenticeship», dont le but est de promouvoir des situations authentiques d'apprentissage à l'école, afin de favoriser le transfert des connaissances scolaires aux situations quotidiennes. Comme le souligne Allal (2001), l'authenticité d'une activité ne se réfere pas tant au fait que l'activité revêt un habillage didactique prenant appui sur un contexte de la vie courante, mais elle est davantage fonction de la nature des dynamiques interactives et de l'usage des ressources sociales et matérielles à disposition dans les situations. En ce sens, l'étude des structures de participation délimitées par les normes de la microculture de classe constitue une entrée pour interroger l'authenticité des processus d'enseignement et d'apprentissage contextualisés dans une communauté classe.

$\mathrm{Si}$, dans cette optique, ce sont les processus sociaux qui sont plus spécialement examinés, les résultats de recherche présentés dans cet article révèlent de façon manifeste des différences cognitives inter-individuelles qu'il semble difficile d'ignorer. Bien que les dyades aient toutes deux développé une procédure ma- 
thématique fondée principalement sur une résolution par isomorphisme de mesures (Vergnaud, 1981, 1988) concernant la relation multiplicative principale en jeu, on observe un niveau d'élaboration différent des procédures déployées, et des façons contrastées d'interpréter et de mettre en relation les données du problème mathématique. Dans les travaux sur la microculture de classe, qui ont fourni un cadre conceptuel à l'analyse des structures de participation observées, les dimensions individuelles et collectives de l'activité mathématique sont conçues dans une relation de réflexivité au sens fort. Les processus sociaux et psychologiques sont vus comme se constituant mutuellement, sans rapport de subordination, et ne pouvant exister les uns sans les autres (e.g., Cobb, Gravemeijer, Yackel, McClain \& Whitenack, 1997 ; Cobb, Perlwitz, Underwood, 1994). Dans la suite de nos travaux et dans l'optique de cette perspective non dualiste, il conviendrait donc de questionner en quoi les processus de constitution et d'actualisation des normes ${ }^{8}$ contraignent et, tout à la fois, rendent possible l'activité cognitive des élèves. Et, réciproquement, il s'agirait d'examiner la façon dont l'activité cognitive individuelle contraint et permet le développement des processus interpersonnels et communautaires de la microculture de classe.

\section{Remerciements}

Mes chaleureux remerciements à Linda Allal pour nos discussions sur la version initiale de l'article.

\section{Notes}

1 Dans la suite de l'article, j'emprunterai la terminologie proposée par Greeno (1997) en parlant de "perspectives situées» pour désigner de façon générale ce courant qui se réfere à une large collection de travaux scientifiques.

2 Cette leçon s'inscrit dans une suite de séances observées sur plusieurs mois dans le cadre d'une étude pilote d'un travail de thèse (Mottier Lopez, 2001).

3 Les auteurs utilisent le concept taken-as-shared pour désigner le fait que les participants interagissent comme s'ils partageaient une même signification d'un objet, bien qu'à titre individuel des différences d'interprétation et de compréhension puissent exister (Cobb \& Bauersfeld, 1995).

4 L'analyse a priori est présentée de façon plus détaillée dans Mottier Lopez (2002).

5 Ce que nous appellerons plus tard «valeur unitaire» de la relation multiplicative «nombre de jours» - «nombre de pommes».

6 L'expression «se sentir obligé» n'a pas une connotation négative, mais a pour but de mettre en évidence une obligation relative aux activités en groupes du point de vue de l'élève.

7 Cette hypothèse interprétative demanderait à être confirmée sur un plus grand nombre de leçons.

8 Entre autres éléments évidemment.

\section{Références bibliographiques}

Allal, L. (1988). Vers un élargissement de la pédagogie de maitrise: processus de régulation interactive, rétroactive et proactive. In M. Huberman (Ed.), Assurer la réussite des apprentissages scolaires? Les propositions de la pédagogie de maîtrise (pp. 86-126). Neuchâtel: Delachaux et Niestlé. 
Allal, L. (2001). Situated cognition and learning: from conceptual frameworks to classroom investigations. Revue Suisse des Sciences de l'éducation, 3, 407-420.

Allal, L., Bétrix Köhler, D., Rieben, L., Rouiller, Y., Saada-Robert, M. \& Wegmuller, E. (2001). Conceptions du curriculum, séquences didactiques et processus d'apprentissage, Apprendre l'orthographe en produisant des textes (pp. 16-42). Fribourg: Editions Universitaires Fribourg Suisse.

Baeriswyl, F. \& Thévenaz, T. (2001). Editorial, Etat des lieux et perspective de la cognition et de l'apprentissage situés. Revue suisse des sciences de l'éducation, 23, 395-405.

Balacheff, N. (1988). Le contrat et la coutume, deux registres des interactions didactiques. In C. Laborde (Ed.), Actes du premier colloque Franco-Allemand de didactique des mathématiques et de l'informatique (pp. 15-26). Grenoble: La pensée sauvage.

Brousseau, G. (1986). Fondements et méthodes de la didactique des mathématiques. Recherches en didactiques des mathématiques, 7, (2), 33-115.

Brown, J.S., Collins, A. \& Duguid, P. (1989). Situated cognition and the culture of learning. Educational Researcher, 18, (1), 32-42.

Cobb, P. (1995). Mathematical learning and small-group interactions: four case studies. In P. Cobb \& H. Bauersfeld (Ed.), The emergence of mathematical meaning (pp. 25-130). Hillsdale NJ: Lawrence Erlbaum Associates Publishers.

Cobb, P. \& Bauersfeld, H. (Ed.). (1995). The emergence of mathematical meaning. Interaction in classroom cultures. Hillsdale, NJ: Lawrence Erlbaum Associates Publishers.

Cobb, P., Perlwitz, M. \& Underwood, D. (1994). Construction individuelle, acculturation mathématique et communauté scolaire. Revue des sciences de l'éducation, 10, 41-61.

Cobb, P., Stephan, M., McClain, K. \& Gravemeijer, K. (2001). Participating in classroom mathematical practices. Journal of the Learning Sciences, 10, 113-164.

Cobb, P., Gravemeijer, K., Yackel, E., McClain, K. \& Whitenack, J. (1997). Mathematizing and symbolizing: The emergence of chains of signification in one first-grade classroom. In D. Kirshner \& J. A. Whitson (Ed.), Situated cognition, social, semiotic, and psychological perspectives (pp. 151-233). Mahwah: Lawrence Erlbaum Associates Publishers.

Collins, A., Brown, J.S. \& Newman, S.E. (1989). Cognitive apprenticeship: Teaching the craft of reading, writing, and mathematics. In L.S. Resnick (Ed.), Knowing, learning, and instruction (pp. 449-453). Hillsdale, NJ: Erlbaum.

Danalet, C., Dumas, J.P., Studer, C. \& Villars-Kneubühler, F. (1999). Mathématiques 4P: livre de l'élève. Neuchâtel: COROME; Berne: Editions scolaires.

Erickson, F. (1986). Qualitative methods in research on teaching. In M.C. Merlin (Ed.), Handboock of research on teaching (pp. 119-161). New York: Macmillan Publishing Company.

Gilly, M., Fraisse, J. \& Roux, J.-P. (1988). Résolution de problèmes en dyades et progrès cognitifs chez des enfants de 11 à 13 ans: dynamiques interactives et socio-cognitives. In A.N. Perret-Clermont \& M. Nicolet (Ed.), Interagir et connaître, enjeux et régulations sociales dans le développement cognitif (pp. 73-92). Cousset Fribourg: Editions DelVal.

Greeno, J.G. (1997). On claims that answer the wrong questions. Educational Researcher, 26, (1), 5-17.

Lampert, M. (1990). When the problem is not the question and the solution is not the answer: Mathematical knowing and teaching. American Educational Research Journal, 27, (1), 29-63.

Lave, J. (1988). Cognition in practice: Mind, mathematics and culture in everyday life. Cambridge: Cambridge University Press.

Lave, J. \& Wenger, E. (1991). Situated learning: Legitimate peripheral participation. Cambridge: Cambridge University Press.

Moll, L.C. \& Whitmore, K.F. (1993). Vygotsky in classroom practice: Moving from individual transmission to social transaction. In E.A. Forman, N. Minick \& C.A. Stone (Ed.), Contexts for learning, sociocultural dynamics in children's development (pp.19-42). New York: Oxford University Press. 
Mottier Lopez, L. (1999). Evaluation formative des apprentissages en mathématiques à l'école primaire. Mémoire de licence, Université de Genève, Faculté de psychologie et des sciences de l'éducation.

Mottier Lopez, L. (2001). Linteraction collective dans la classe de mathématiques: observation de la participation aux pratiques sociales de la communauté classe en 3ème année primaire. Canevas de thèse en sciences de l'éducation, Université de Genève.

Mottier Lopez, L. (2002). Problème mathématique «Une pomme pour la récré»: Gros plan sur l'interprétation et le raisonnement mathématiques de quatre élèves. Math-Ecole, 204, 3048.

Nunès, T. (1991). Systèmes alternatifs de connaissances selon différents environnements. In C. Garnier, N. Bednarz \& I. Ulanovskaya (Ed.), Après Vygotsky et Piaget. Perspectives sociale et constructiviste. Ecoles russe et occidentale (pp. 117-128). Bruxelles: De Boeck université.

Pourtois, J.P. \& Desmet, H. (1997). Epistémologie et instrumentation en sciences humaines (2e éd.). Sprimont: Maradaga.

Resnick, L.B. (1987). The 1987 Presidential Address, Learning In school and Out. Educational Researcher, 16, (9), 13-20.

Resnick, L.B., Pontecorvo, C. \& Säljo, R. (1997). Discourse, Tools, and Reasoning: Essays on Situated Cognition. Berlin: Springer.

Rogoff, B. (1995). Observing sociocultural activity on three planes: participatory appropriation, guided participation, and apprenticeship. In J.V. Wertsch, P. del Rio \& A. Alvarez (Ed.), Sociocultural studies of mind (pp. 139-164). Cambridge: Cambridge University Press.

Schubauer-Leoni, M.L. (1986). Le contrat didactique: un cadre interprétatif pour comprendre les savoirs manifestés par les élèves en mathématiques. Journal européen de psychologie de l'éducation, 2, 139-153.

Stake, R. (1995). The art of case study research. Thousand Oaks, CA: Sage Publications.

Vergnaud, G. (1981). L'enfant, la mathématique et la réalité. Berne: Peter Lang.

Vergnaud, G. (1988). Multiplicative structures. In J. Hiebert \& M. Behr (Ed.), Number concepts and operations in the middle grades (pp. 141-161). Reston VA: National Council of Teachers of Mathematics, Lawrence Erlbaum Associates.

Voigt, J. (1985). Pattern and routines in classroom interaction. Recherches en didactique des mathématiques, 6, (1), 69-118.

Voigt, J. (1995). Thematic patterns of interaction and sociomathematical norms. In P. Cobb $\& \mathrm{H}$. Bauersfeld (Ed.), The emergence of mathematical meaning: Interaction in classroom cultures (pp. 163-202). Hillsdale, NJ: Lawrence Erlbaum Associates publishers.

Yackel, E. \& Cobb, P. (1996). Sociomathematical norms, argumentation and autonomy in mathematics. Journal for Research in Mathematics Education, 27, 458-471.

\section{Strukturen der Beteiligung an der Mikrokultur der Klasse in einer Mathematikstunde}

\section{Zusammenfassung}

Sich an die theoretischen Vorschläge des situierten Lernens anlehnend stellt dieser Beitrag eine Detailstudie über eine Mathematikstunde, deren Ablauf durch verschiedene soziale Organisationsformen strukturiert war, im vierten Primarschuljahr vor. Das Ziel ist, ausgehend von der Analyse der interpersonalen Prozesse zwischen den Schülern und mit dem Lehrer den gemeinsamen Plan der Klasse zu untersuchen, indem die Miterarbeitung der Interpretation und der 
mathematischen Argumentation bei einem komplexen Multiplikationsproblem analysiert wird. Die Forschungsergebnisse zeigen einerseits, dass die gleiche Norm, die für eine in der Mikrokultur der Klasse hoch bewertete Beteiligungsstruktur steht, die didaktischen Interaktionen sowohl in der kollektiven Interaktion als auch in den Gruppenarbeiten strukturieren kann. Andererseits wird gezeigt, dass die Aktualisierung dieser Norm in kontrastreichen Formen der Beteiligung zwischen den Schülern erfolgt.

\section{Le strutture di partecipazione a livello di microcultura di classe in una lezione di matematica}

\section{Riassunto}

Muovendo dalle premesse teoriche dell'apprendimento situato, l'articolo analizza in modo dettagliato una lezione di matematica in una quarta elementare strutturata sulla base di diverse forme sociali di lavoro. L'obiettivo è quello di mettere in discussione l'organizzazione sociale della classe partendo dall'analisi dei processi interpersonali fra gli allievi e con l'insegnante ed esplorando l'elaborazione comune dell'interpretazione e del ragionamento matematico riferito ad un problema di moltiplicazione. I risultati mostrano che la stessa norma rappresentazionale delle strutture participative, valorizzata nella microcultura della classe, può informare le interazioni didattiche sia collettive sia nell'ambito dei gruppi di lavoro degli allievi. Si mette pure in evidenza l'attuazione di questa norma in forme di co-elaborazione caratterizzate da contrasti.

\section{The participation structures of the classroom microculture in a mathematics lesson}

\section{Summary}

On the basis of the conceptual framework of situated learning, the article presents a detailed study of a fourth-grade mathematics lesson, which is structured by various forms of social organization. The purpose is to elucidate the community dimension of the classroom based on the analysis of interpersonal processes, among the pupils and with the teacher, by examining the co-elaboration of the mathematical interpretation and reasoning regarding a multiplication problem. The results of the study show that a given norm representative of the participation structures valued in the classroom microculture appears to structure didactic interactions during both the collective interaction and the small group work. In addition, the manifestations of this norm in contrasted forms of co-elaboration among pupils are identified. 